\title{
Offshore LoRaWAN Networking: Transmission Performances Analysis Under Different Environmental Conditions
}

This is the peer reviewed version of the following article:

Original:

Parri, L., Parrino, S., Peruzzi, G., Pozzebon, A. (2021). Offshore LoRaWAN Networking: Transmission Performances Analysis Under Different Environmental Conditions. IEEE TRANSACTIONS ON INSTRUMENTATION AND MEASUREMENT, 70, 1-1 [10.1109/TIM.2020.3031193].

Availability:

This version is availablehttp://hdl.handle.net/11365/1121718

since 2020-12-06T12:23:25Z

\section{Published:}

DOI:10.1109/TIM.2020.3031193

Terms of use:

\section{Open Access}

The terms and conditions for the reuse of this version of the manuscript are specified in the publishing policy. Works made available under a Creative Commons license can be used according to the terms and conditions of said license.

For all terms of use and more information see the publisher's website.

(Article begins on next page) 


\title{
Offshore LoRaWAN Networking: Transmission Performances Analysis Under Different Environmental Conditions
}

\author{
Lorenzo Parri, Stefano Parrino, Giacomo Peruzzi and Alessandro Pozzebon, Member, IEEE.
}

\begin{abstract}
This paper presents the architecture and the performances of a Long Range Wide Area Network (LoRaWAN) infrastructure used for data transmission from a floating sensor node placed in the middle of the sea to a multi-Gateway structure positioned on the coast, whose purpose is the monitoring of offshore breeding cages within a fish farming plant. In particular, the sensor node is installed on a seamark buoy and it is interfaced with ad hoc sensors for the measurement of marine parameters. All sampled data are conveyed ashore to the Gateways by successfully covering the distance of $8.33 \mathrm{~km}$. The paper presents the results concerning the performances of the data transmission for a 70 days operating period, analyzing the radio parameters (Signal-to-Noise Ratio - SNR and Received Signal Strength Indicator - RSSI) in relation with the variations of environmental parameters like temperature, relative humidity and atmospheric pressure as well as weather conditions. The proposed system demonstrates the usability of the LoRaWAN in all those cases where data collection from offshore monitoring structures is required: while this infrastructure focuses on the monitoring of fish farming plants, it may find application in several contexts, from navigational buoys to offshore oil plants.
\end{abstract}

Index Terms-Fish Farming, LoRaWAN, Industry 4.0, Transmission Performances Analysis, Environmental Parameters.

\section{INTRODUCTION}

$\mathbf{L}$ ONG Range (LoRa) technology, together with the associated Long Range Wide Area Network (LoRaWAN) protocol, is currently one of the most promising data transmission technologies within the Internet of Things (IoT) domain for what concerns the so-called Sub-GHz systems (i.e., communication technologies operating at frequencies below $1 \mathrm{GHz}$ ). Its success comes from the fulfillment of three requirements that can be crucial when designing an IoT infrastructure: the long transmission range, the low power consumption and the low cost of the devices as well as of the overall network infrastructure. For this reason, in the last years LoRaWAN systems have been employed in a wide range of applications, also in critical scenarios where the technical features of this technology allow to overcome limitations like, for example, the harshness of the deployment site of the data acquisition system. In this context, the use of LoRaWAN has been also tested for the data acquisition in coastal areas, for example for the monitoring of offshore structures like fish farming sites. Indeed, fish farming is a worldwide booming economy: a forecast on global seafood market in 2030 [1] estimates

L. Parri, S. Parrino, G. Peruzzi and A. Pozzebon are with the Department of Information Engineering and Mathematics, University of Siena, Siena, 53100 Italy, e-mail: parri@diism.unisi.it,parrino2@unisi.it, peruzzi@diism.unisi.it, alessandro.pozzebon@unisi.it. that $62 \%$ of fish for human consumption will be produced via aquaculture by that year. Moreover, in 2014 the Food and Agriculture Organization (FAO) predicted an optimistic scenario which assumed an aquaculture production increase of $58 \%$ by 2022 [2].

Together with the fish farming scenario, LoRaWAN infrastructures may be used in several other contexts for data transmission from offshore sites to ashore data management systems: examples include environmental floating monitoring stations acquiring marine and environmental parameters, monitoring infrastructures deployed on near-shore islands or even data acquisition devices placed on offshore oil plants.

Nevertheless, an offshore data transmission system has to satisfy more severe system requirements in comparison with an inland-deployed one. Indeed, the sea surface itself attenuates the transmitted signal, thus reducing the transmission range. In this context, the feasibility of a LoRa link was beforehand validated within previous works [3], [4]. In particular this paper extends [4], where preliminary results were reported, by showing the fully operating system and the medium term measurement campaign.

At the same time, offshore transmitting devices, as well as any instrument positioned in an outdoor environment, are exposed to environmental agents that may degrade their performances or unable data transmission in certain weather conditions. For this reason, in order to fully validate the operation of a system and push its adoption in real applications, its functioning should be demonstrated in a long term period and in a wide range of different environmental conditions. Nevertheless, while some works can be found in literature analyzing the performances of LoRaWAN systems for what concerns the transmission range and the behavior in non lineof-sight conditions and in presence of obstacles, as far as we are concerned no work has been found discussing the performances of the LoRa technology in different environmental and meteorological conditions. Similarly, no work has been found analyzing the system operation for prolonged spans of time.

The aim of this paper is then to demonstrate the feasibility of a LoRaWAN infrastructure for offshore data transmission, analyzing the behavior of the system for a prolonged period (i.e., 70 days), correlating the transmission performances with environmental and meteorological parameters related to the deployment site. For this reason, an offshore sensor node was installed on board of a seamark buoy deployed in the middle of a sea farming plant composed of 24 cages, positioned 8.33 $\mathrm{km}$ offshore the city of Piombino, Italy. This node is in charge 
of acquiring samples from ad hoc sensors so to monitor the state of either the bred fish and the breeding cages. Such data is ashore conveyed, by means of LoRa transmissions, where two LoRaWAN gateways are setup. The latter ones forward the gathered information towards remote servers by availing of the Message Queue Telemetry Transport (MQTT) protocol.

The rest of this paper is arranged as follows. Some related works analyzing the performances of LoRaWAN systems, either in the marine environment and in other use cases, are reported in Section II. The operating scenario our network infrastructure belongs to and its overall description are exposed in Section III while the design and implementation of the system are delineated in Section IV. Section V shows the network prototype testing procedures and their results while Section VI treats the results discussion. Finally, Section VII underlines conclusions and remarks.

\section{RELATED WORKS}

LoRa technology and LoRaWAN protocol have seen a vast diffusion in the last years, thanks to the their features in terms of long range data transmission and low power consumption, and have been employed to set up a wide range of distributed monitoring systems in different application fields, from industry [5] and agriculture [6] to the well-known Smart City scenario [7]. Environmental parameters have been sampled for decades for the most diverse scopes, particularly for the analysis of climatic and ecological changes, and the adoption of LoRaWAN within this field brought about several advantages [8]-[12].

Due to its importance as an enabling technology in a wealth of applications, several works have focused on the analysis of the performances of the LoRa technology. In general, since the two most interesting technical features of LoRa systems are its long transmission range and low power consumption, the bulk of these papers focuses on the evaluation of the maximum achievable transmission distance evaluating the system performances in terms of Received Signal Strength Indicator (RSSI) and Signal to Noise Ratio (SNR). Other parameters that are evaluated include Packet Delivery Rate (PDR) (or, in a complementary way Packet Loss (PL)), Time on Air (ToA), bitrate and delay, even if these last three are rarely taken into account since LoRa is intrinsically a high-latency, low-bitrate technology: this means that it is generally employed in those applications (e.g., the one discussed in this paper) where latency and throughput are not crucial. All the aforementioned parameters are usually evaluated combining the different configurable LoRa settings, i.e., Transmission Power, Coding Rate (CR), Bandwidth (BW) and Spreading Factor (SF): this last parameter is in general the one that receives the largest attention since is the one that has more influence on system performances in terms of achievable transmission range.

Performances are mostly evaluated according to the features of the deployment scenario: for example, Cattani et al. [13] evaluate PDR and bitrate in three different settings (i.e., indoor, outdoor and underground) combining different LoRa parameters, while Sanchez-Iborra et al. [14] analyze the theoretical coverage of LoRa in urban, suburban and rural scenarios at different SFs. Other works concentrate in detail on a single scenario. PL and RSSI for different SFs in an indoor environment are discussed in [15], while performances in a larger area like a university campus are discussed in [16] by taking into account RSSI and delay in Line-of-Sight and Non-Line-of-Sight. Conversely, [17] investigates indoor and outdoor PL. Similarly, Haxhibeqiri et al. [18], analyze indoor and outdoor RSSI and SNR in an industrial environment changing the SF, simulating the performances in terms of PL for a large number of sensor nodes: this is a feature that can be hardly evaluated by means of field tests due to the need of thousands of transmitting devices. Widening the scale of the testing site, performances in urban areas are evaluated in [19] (i.e., RSSI, SNR and PL) and [20] (i.e., RSSI, PDR and Throughput), in both cases for different SFs, while Yim et al. [21] analyze PDR and RSSI in a rural area within a smart agriculture application, testing different configurations, combining different SFs, BWs and CRs. While all those papers prove the effectiveness of the LoRa link, only in [13] the correlation among temperature and RSSI is analyzed, while in [17] the influence of weather on system performances is taken into account across a 24-hours timespan. Nevertheless, no work proposes a detailed analysis of the effects of different environmental and meteorological conditions on the LoRa performances. Similarly, no long term test is carried out, in particular in harsh environments like the marine one.

Indeed, marine environments are extremely hostile thus hindering either the maintenance of assets overboard installed as well as the measurement of environmental parameters. Therefore, LPWANs are advisable for the optimization of the aforesaid procedures. Among all the enabling technologies, LoRa modulation and LoRaWAN protocol confirmed to be the most suitable ones especially due to their robustness to interference, which naturally comes into play in a marine environment, and their limited running costs [22]-[26]. However, LoRa-based full network infrastructures to be deployed offshore and, in general, LoRa transmissions over seawater are still hot research topics nowadays thus entailing a lack of related works within the literature. However, concerning LoRa links over the sea, or in general over water basins, some works proved their practicability: from the monitoring of brine pumps within saline lakes [27] to the assessment of potential tidal energy in sites at risk [28], or from the tracking of drifters [29] to the testing of LoRa modulation [30] and LoRaWAN protocol [3], [4] forthwith on the field.

\section{OPERATING SCENARIO AND PROPOSED SOLUTION}

The LoRaWAN network prototype presented in this paper was specially designed in order to fulfill the objectives of SeaFactory, a strategic research and development project, funded by the Tuscany Region, Italy, whose goal is to aid fish farming companies in the migration towards an Industry 4.0 paradigm. For this aim, a prototype of an operational control centre for the management of a variety of goods, located both on land and sea, (e.g., infrastructures, transformation plants, instruments and so on) was established. To this end, a 


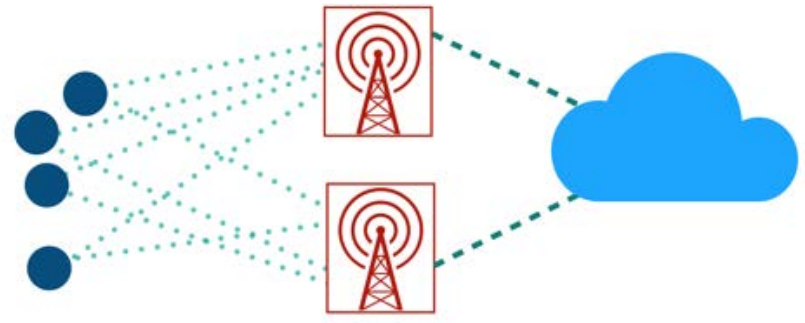

\section{Sensor Nodes LoRa Gateways MQTT Servers}

Fig. 1. Block diagram of the overall solution architecture.

prototype of LoRaWAN network architecture was devised. It includes:

- End Nodes, that are installed on board of seamark buoys which are in charge of offshore collecting data and wirelessly transmit them towards land via encrypted LoRa packets exploiting a frequency hopping scheme as LoRaWAN protocol prescribes. The measurements are accomplished by means of suitable sensors that are provided by a project partner firm. Those data are needed to assess the environmental conditions of breeding;

- Gateways, which are responsible for the demodulation of the signals and for the forwarding of the received information to remote Servers by making use of the MQTT protocol;

- Network Server, that deals with the incoming packets by processing and storing them in order to extract any useful data;

- Application Server, which makes data available to users via a graphic interface.

Hence, a LoRaWAN network was selected since it well suits for the application scenario due to its low cost, long range and low power features which are strongly required for this particular project. The aforementioned solution and technologies are summarised in Fig. 1. For the sake of completeness, several solutions worked out by third parts implementing full LoRaWAN facilities already exist. However, those alternatives were discarded since SeaFactory requires a flexible and bespoke network infrastructure. In addition, all the gathered data have not to be divulged thus reinforcing the necessity to build up a customised back-end.

Aquaculture companies may benefit from this type of monitoring systems. Indeed, a wireless connectivity to offshore plants is provided thus allowing the reduction of their inspections by personnel along with the entailed costs. On the other hand, a system like this needs to be carefully conceived so to withstand to the harshness of maritime environment (e.g., waves, salt, seaweed, storms, marine birds, etc.). In addition, each of the monitored physical phenomena requires an ad hoc sensor or probe which has to suit to the environment. That is the reason why specially-made marine probes are employed so to supply the aquaculture firm with marinerelated data (e.g., underwater temperature, speed and direction of sea currents, etc.). In doing so, breeding phases may be thoroughly optimized. In a near future the offshore system may be integrated within a unique device which could make part of the breeding cages so to augment its robustness and effectiveness. Finally, in spite of the fact that this system was devised for offshore seafarms, it may be exploited for the monitoring of any other type of offshore asset (e.g., offshore oil plants) thus highlighting its versatility.

\section{SYSTEM DESIGN AND IMPLEMENTATION}

The LoRaWAN network prototype of Fig. 1 presents some lacks affecting the back-end side that, nevertheless, neither are strictly required for the aforementioned project nor they undermine the network functioning. Indeed, the Network Server only deals with uplinks from End Nodes since there is no need to perform downlinks for this application scenario. For what concerns their activation, only Activation By Personalization (ABP) method is practiced due to the fact that it is the most straightforward. Another feature that is not supported is Adaptive Data Rate (ADR) because it is not advisable for the SeaFactory project since it is deployed in a harsh environment characterized by an unreliable communication channel due to the nature of the marine environment.

Summarizing, this LoRaWAN network prototype is composed by an End Node, two Gateways behaving as packet forwarders, a Network Server and an Application Server both leaning on a shared database.

\section{A. End Node}

At this stage of the project a sensor node is already installed on board of the seamark buoy (see Fig. 2). The End Node is housed within an IP67 box along with a data logger, that is designed by a project partner firm, whose task is to control all the marine probes (that are submerged) to which the End Node is connected to via a serial port. Concerning the latter, it is a tailor-made embedded system (that was named as LoRaNode V1) acting like an RS-232 - LoRaWAN interface (see Fig. 3 ) whose purpose is to arrange data coming from sensors in LoRaWAN packets and to broadcast such information ashore by covering an $8.33 \mathrm{~km}$ distance.

Albeit the seamark buoy hosts two photovoltaic panels, the LoRaNode V1 board is designed in order to be battery powered. Indeed, the panels are solely necessary to supply the sensors probes and the data logger because they are the power hungrier elements. Since the system has to satisfy a several months lifetime, the LoRaNode V1 needs to be very low power, hence the following components were embedded into it: an STM32L073 microcontroller [31] produced by STMicroelectronics, an RFM95 LoRa transceiver [32] manufactured by HopeRF (which relies on an SX1276 LoRa module [33] produced by Semtech) and miscellaneous low power electronics. Finally, the system is powered by a 3600 $\mathrm{mAh}$ Li-ion battery providing $3.7 \mathrm{~V}$. According to the project monitoring requirements, a sampling on a hourly basis is needed. In particular, all the data is split in 3 LoRaWAN packets that are sent 3 times in order to overcome losses which naturally come into play (as previous works pointed out [3], [4]). Concerning the transmission parameters, packets are broadcast exploiting a BW of $125 \mathrm{kHz}$, an $\mathrm{SF}$ of 7 and 


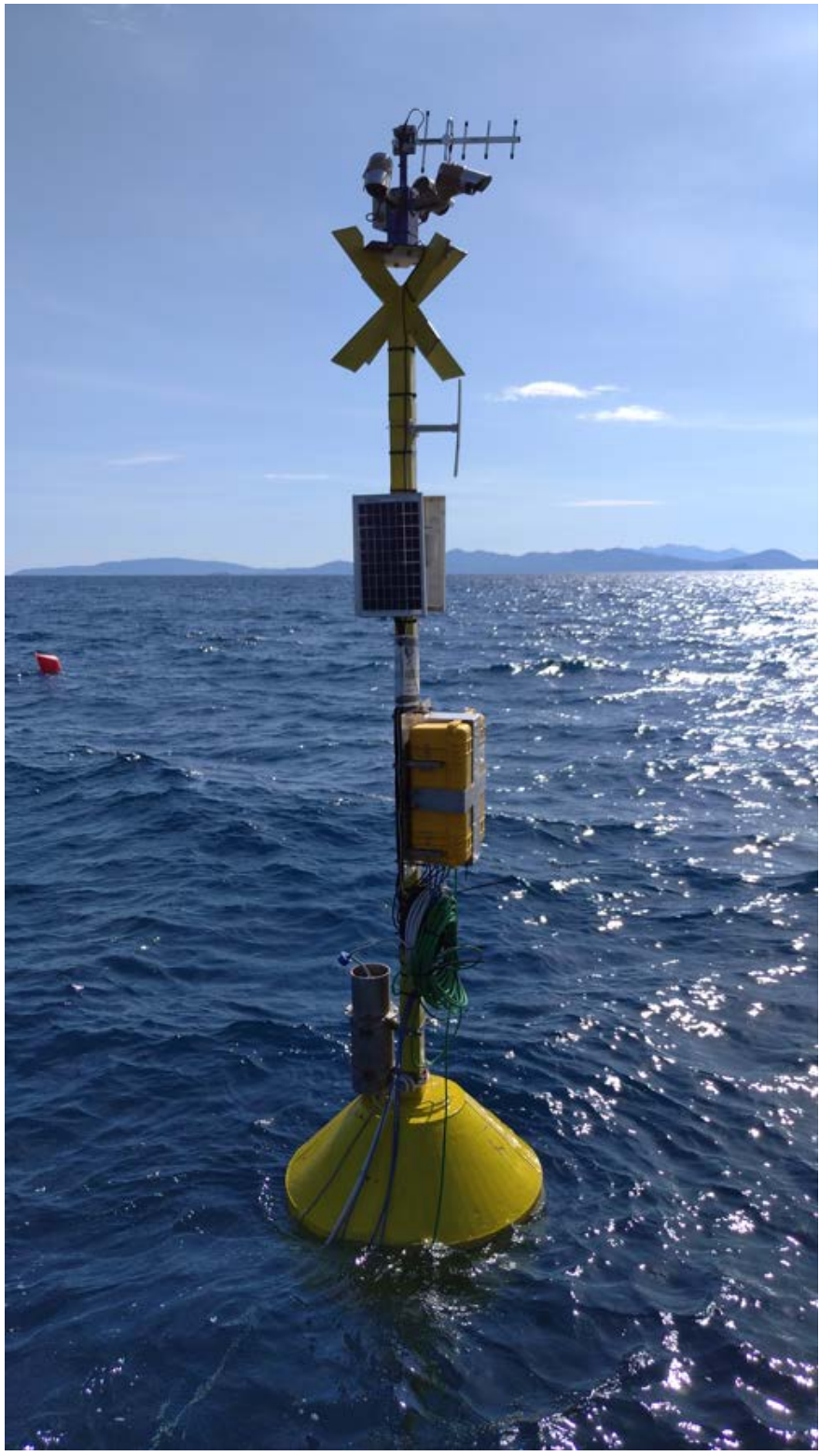

Fig. 2. Seamark buoy on which the sensor node is installed.

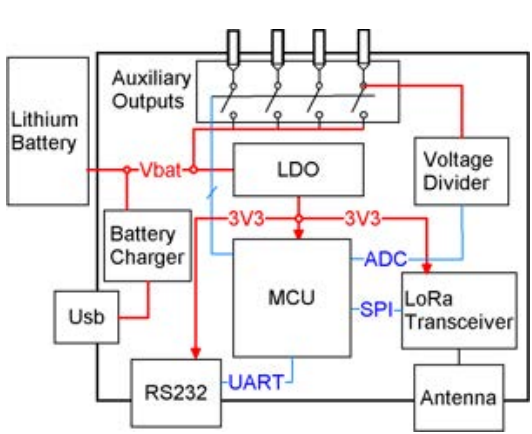

(a)

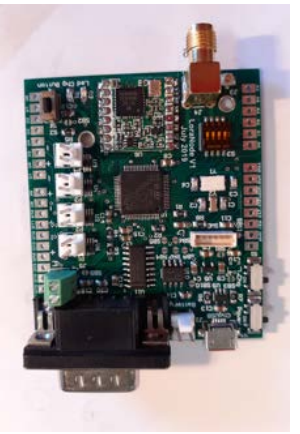

(b)
Fig. 3. LoRaNode V1 embedded system: (a) block diagram and (b) board without battery and antenna.

a CR of $4 / 5$ since, by resorting to results of previous works, such settings proved to be successful while minimizing power consumption. Moreover, the LoRaNode V1 sends packets exploiting a power of $14 \mathrm{dBm}$ and respecting the laws on the temporal occupancy of the exploited Industrial, Scientific and Medical (ISM) band (i.e., devices have to respect a duty cycle of $1 \%$ [34]). The firmware running on the microcontroller implements a LoRaWAN Class A device (i.e., a network End Node). Hence, it encrypts the packet payload twice, by means of the Network Session Key (NwkSKey) and the Application Session Key (AppSKey), exploiting an Advanced Encryption Standard (AES) (i.e., AES-128). Then, such packets are broadcast to any Gateway in the vicinity establishing a frequency diversity scheme via a frequency hopping technique amid 8 different channels belonging to the $863 \div 870 \mathrm{MHz}$ ISM band (i.e., 867.1 MHz, 867.3 MHz, 867.5 MHz, 867.7 MHz, 867.9 $\mathrm{MHz}, 868.1 \mathrm{MHz}, 868.3 \mathrm{MHz}$ and $868.5 \mathrm{MHz}$ ). Finally, a 9 $\mathrm{dBi}$ Yagi-Uda antenna mounted on top of the seamark buoy (i.e., at $2 \mathrm{~m}$ above sea level) is exploited for the transmission. The connection between the antenna and the End Node is established by means of a $4 \mathrm{~m}$ coaxial cable whose loss is $2.10 \mathrm{~dB}$.

\section{B. Gateways}

The block diagram of the LoRaWAN Gateway (i.e., a LoRaWAN Class C device) is depicted in Fig. 4a. The concentrator is a particular board composed of a LoRa modem and some LoRa transceivers: the RAK831 produced by RAKWireless [35] was selected for such purpose. It is a multi-channel high performance concentrator designed to simultaneously receive and demodulate up to $8 \mathrm{LoRa}$ packets that could have been transmitted by using different SFs on multiple channels. Of course, such a knowledge is not available to the concentrator a priori and still it accomplishes that task. The channels the RAK831 listens to are the same the End Node exploits for the transmission. Its principal building blocks are: two SX1257 [36] (i.e., LoRa transceivers) and one SX1301 [37] (i.e., a LoRa modem), both produced by Semtech. According to the concentrator datasheet, by exploiting this hardware architecture the Gateway reaches a sensitivity that varies according to SF and $\mathrm{BW}$ : considering the aforesaid transmission settings, a sensitivity of $-126 \mathrm{dBm}$ is reached. This hints at a potential wide coverage in the radius of tens of kilometers. The concentrator is driven via a Serial Peripheral Interface (SPI) by a Raspberry Pi 3 model B which is a single-board computer whose tasks are: running all the needed software and routines, driving the concentrator and connecting to the Network Server via the Internet by making use of the MQTT protocol. For the sake of redundancy, thus increasing the probability to correctly receive uplinks from the seamark buoy, two identical Gateways were ashore set up (see Fig. 5) in order to establish a space diversity scheme so as to enhance the delivery reliability. Indeed, since Gateways are built by resorting to low cost off-the-shelf components, it is advisable to establish such a redundancy so to overcome losses and possible hardware failures: in the case in which one of the two Gateways goes down, the other one could still fulfil its tasks so to ensure the overall system functionality. Moreover, Gateways are mains 
(a)

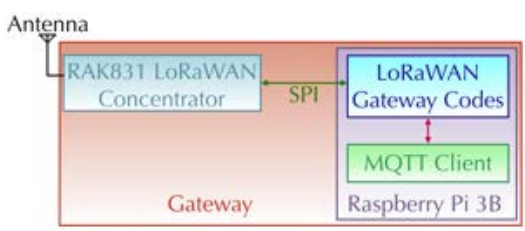

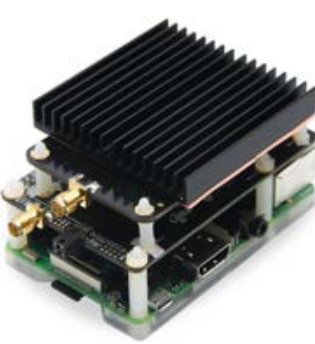

(b)
Fig. 4. LoRaWAN Gateway: (a) block diagram and (b) hardware implementation.

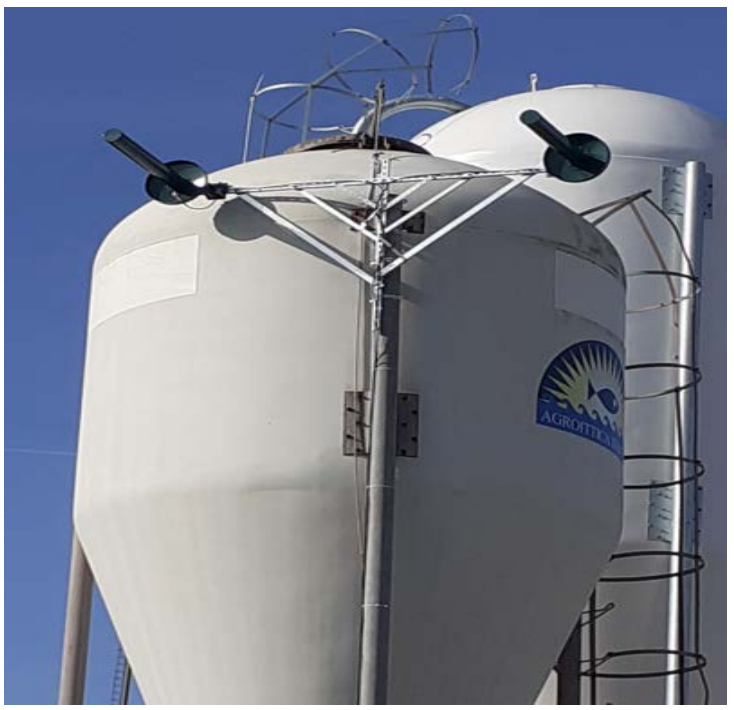

Fig. 5. Gateways and their antennas in the installation site.

powered since they are constantly listening for incoming LoRa packets and they are always connected to the Network Server. All the schematised components in Fig. 4a were physically assembled obtaining the result in Fig. $4 \mathrm{~b}$ that shows a layered structure: the lower one is the Raspberry $\mathrm{Pi}$, the middle one is an adapter connecting the Raspberry $\mathrm{Pi}$ to the upper layer which is the RAK831 concentrator on which a heat sink is mounted.

In addition, the two Gateways are connected to as much directional helical antennas whose gain is $14 \mathrm{dBi}$ each, which are installed at an altitude of $5 \mathrm{~m}$ above sea level. The connection is performed via two $15 \mathrm{~m}$ coaxial cables whose individual loss is $4.06 \mathrm{~dB}$, while Internet connectivity is provided by a $4 \mathrm{G}$ LTE router.

As it was previously said, the Raspberry Pi executes all the codes implementing a LoRaWAN Gateway, which simply behaves as a packet forwarder. The software running on the Raspberry Pi consists of a multi-thread program which is joined to a Python script implementing an MQTT client acting as a publisher and connected to the MQTT broker running on the Network Server. The former demodulates the packets extracting the encrypted payloads and appending to each of them a series of metadata (e.g., RSSI and SNR), while the latter routes all this information to the broker by publishing it on Gateway-specific MQTT topics as MQTT messages with

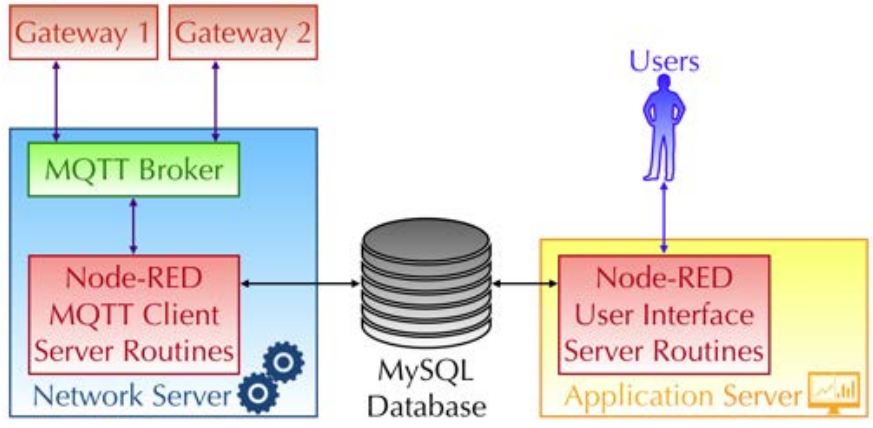

Fig. 6. Complete Server architecture: violet arrows represent the MQTT links while the black ones symbolise the connections to the database. Gateways have been included just for a matter of completeness even though they are not part of the back-end side of the network.

a Quality of Service (QoS) equal to 0 (i.e., a QoS of At Most Once that is also known as fire and forget) so to shun message re-posting thus reducing latency. In the same way, the Gateways send to the Network Server their functioning statistics (e.g., the number of received packets) on a periodic basis with a network diagnosis purpose.

\section{Servers}

The back-end of the LoRaWAN network infrastructure prototype (see Fig. 6) is essentially constituted by a Network Server, an Application Server and a MySQL database on which the Network Server stores all the incoming data from End Node and Gateways and from which the Application Server retrieves information. Either the software of the Network Server and the one of the Application Server are implemented by means of Node-RED: a development environment created by IBM, which runs on Node.js, that is based on a flow programming language whose building blocks are written in JavaScript. Some of those nodes are pre-installed (e.g., MQTT clients, debuggers, connections to databases and so on) but personalised JavaScript functions may be created by setting up fully customizable blocks. Node.js is a JavaScript runtime environment that executes JavaScript snippets outside a browser thus enabling their use for scripting on server side.

1) Network Server: The Network Server hosts an MQTT broker and a Node-RED flow implementing all the Server routines:

- Sending acknowledgement signals back to the Gateways via MQTT whenever the MQTT clients running on the latter ones publish messages towards the client of the Server so to accomplish network diagnostic;

- Storing within the MySQL database the Gateways functioning statistics;

- Filtering out duplicated packets forwarded by both the Gateways rejecting those having the worst RSSI;

- Decrypting the End Nodes packets payloads by making use of the NwkSKey and of the AppSKey which are contained in the MySQL database;

- Discarding packets that were sent by End Nodes belonging to other networks by performing the Message Integrity Code (MIC) check. Indeed, any End Node that is 
part of other LoRaWAN networks encrypts payloads with diverse NwkSKey and AppSKey from the ones stored in the MySQL database and whenever their payload will be decrypted making use of different AES keys (i.e., the one of this network prototype), an invalid MIC will be observed. So, the Network Server will filter those packets;

- Storing within the MySQL database the decrypted packets payloads, and their associated metadata, that passed the MIC check.

2) Application Server: The Application Server is implemented by a Node-RED flow that extracts data from the MySQL database and exposes them to users via a graphic interface which is remotely consulted via an Internet browser. The interface is organized in tabs, each of which outlines data and metadata related to End Nodes as well as Gateways functioning statistics by means of tables, widgets and plots.

\section{Transmission system costs and power consumption}

The transmission system was developed by pursuing low power and low cost features. The production cost of the LoRaNode V1 is of $30 €$, while its battery $4 €$ and the YagiUda antenna $26 €$. On the other hand, the ashore Gateways reach an overall sum of $540 €$. Hence, a total amount of $600 €$ is obtained which may be too much in order to label the whole system as low cost. However, either the LoRaNode V1 and each of the Gateways are only at their prototypical stage and far from being mass production devices. Hence, a cost reduction is reasonable during future enhancement and improvement of the system.

Power consumption was evaluated only for the LoRaNode V1 board since the Gateways are mains powered. In so doing, an estimate of the battery lifetime can be computed. As it will be explained in the next Section, a working cycle of the board may be roughly split into two phases: the former in which data is processed and broadcast, which demands an energy of $1 \mathrm{mWh}$, and the latter in which the board enters in sleep mode, requiring $0.3 \mathrm{mWh}$. The battery powering the board stores an energy of $13320 \mathrm{mWh}$, thus it can approximately ensure 10200 hourly working cycles that correspond to 425 days.

\section{EXPERIMENTAL SETUP AND TESTS}

The seamark buoy was launched on the $28^{t h}$ May 2020 in point A of Fig. 7, while the ashore Gateways were installed on the $3^{\text {rd }}$ July 2020 in point B of Fig. 7. As a consequence, in this paper 70 days of measurements are reported (i.e., from the $3^{\text {rd }}$ July 2020 13:29 CET to the $10^{\text {th }}$ September 2020 12:34 CET).

According to system requirements concerning avoiding data compression, sensors measurements are arranged in 3 LoRaWAN packets whose payloads have a variable size: one of $51 \mathrm{~B}$ and the remainder two of $106 \mathrm{~B}$. Such a choice is motivated by the fact that regional regulation on the exploitation of ISM bands [34] do not allow to sort out all the measurements only within 2 packets since their payload would be too long. Moreover, in order to reduce the probability of measurements loss, those 3 packets are sent 3 times so to

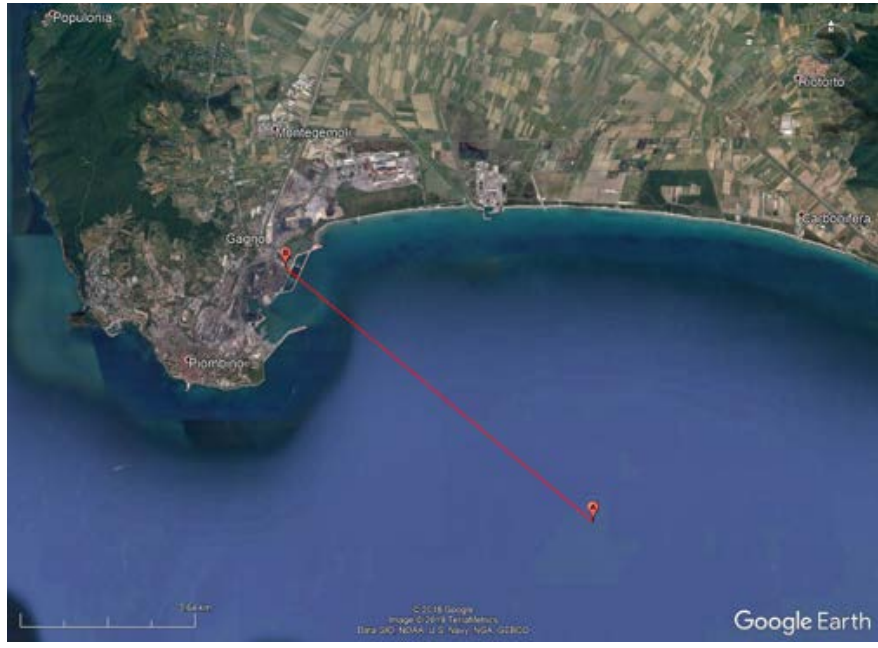

Fig. 7. Map exposing the transmission tests. Point A represents the transmitter and point $\mathrm{B}$ the receivers while the red line depicts the covered distance.

establish a redundancy paradigm. Therefore, the ashore system operates according to the following scheme:

1) The LoRaNode V1 retrieves the samples from the data logger managing the sensors;

2) The LoRaNode V1 arranges the data within 3 LoRaWAN packets;

3) The LoRaNode V1 broadcasts the packets 3 times;

4) The LoRaNode V1 enters in sleep mode until new measurements are sampled by sensor nodes and then it restarts the cycle from step 1.

Such a procedure takes an amount of time of 62 minutes and 51 seconds (i.e., 2 minutes and 44 seconds for accomplishing steps 1 to 3 since packets are sent abiding by regional regulations on temporal occupancy of ISM bands, while 60 minutes and 7 seconds are spent in step 4). Hence, according to the aforesaid procedure, during 70 days of tests 14220 packets were transmitted from the ashore buoy.

The first instance of system performances is the overall number of received packets out of the transmitted ones which is 10301 out of 14220 (i.e., $72.44 \%$ ). In addition, the number of received packets for each of the channels the LoRaNode V1 exploits for performing frequency diversity is evaluated and the results are reported in Table I. Finally, mean values and standard deviations for RSSIs and SNRs are taken into account. In particular, they are firstly computed either for the whole amount of data and for data split by date, and then they are evaluated by dividing data according to weather conditions as well as to atmospheric temperature, relative humidity and atmospheric pressure. Indeed, all of the meteorological information were associated to each of the received packets by resorting to the Application Program Interface (API) that is offered by OpenWeather [38]. The results related to RSSIs and SNRs concerning all of these cases are reported in Table II and in Fig. 8 - 11.

\section{DISCUSSION}

According to the collected data throughout the 70-days measurement campaign, interesting considerations about the 
TABLE I

NUMBER OF RECEIVED PACKETS FOR EACH OF THE CHANNELS.

\begin{tabular}{cc}
\hline Channel $[\mathrm{MHz}]$ & Received Packets \\
\hline 867.1 & 1466 \\
867.3 & 1449 \\
867.5 & 287 \\
867.7 & 1454 \\
867.9 & 887 \\
868.1 & 1901 \\
868.3 & 1426 \\
868.5 & 1431 \\
\hline
\end{tabular}

TABLE II

MEAN VALUES AND STANDARD DEVIATIONS OF RSSIS AND SNRS.

\begin{tabular}{ccccc}
\hline & \multicolumn{2}{c}{ RSSI $[d B m]$} & \multicolumn{2}{c}{ SNR $[d B]$} \\
& $\bar{x}$ & $\bar{s}$ & $\bar{x}$ & $\bar{s}$ \\
\hline Total & -114.33 & 2.33 & -1.34 & 2.53 \\
Clear Sky & -114.24 & 2.32 & -1.30 & 2.58 \\
Few Clouds & -114.45 & 2.29 & -1.48 & 2.48 \\
Scattered Clouds & -114.45 & 2.39 & -1.34 & 2.50 \\
Cloudy & -114.41 & 2.24 & -1.18 & 2.32 \\
Light Rain & -114.97 & 2.17 & -1.90 & 1.98 \\
Moderate Rain & -115.48 & 1.93 & -2.57 & 1.65 \\
Heavy Rain & -116.83 & 1.47 & -2.42 & 2.03 \\
Fog & -113.88 & 2.64 & -2.58 & 3.09 \\
\hline
\end{tabular}
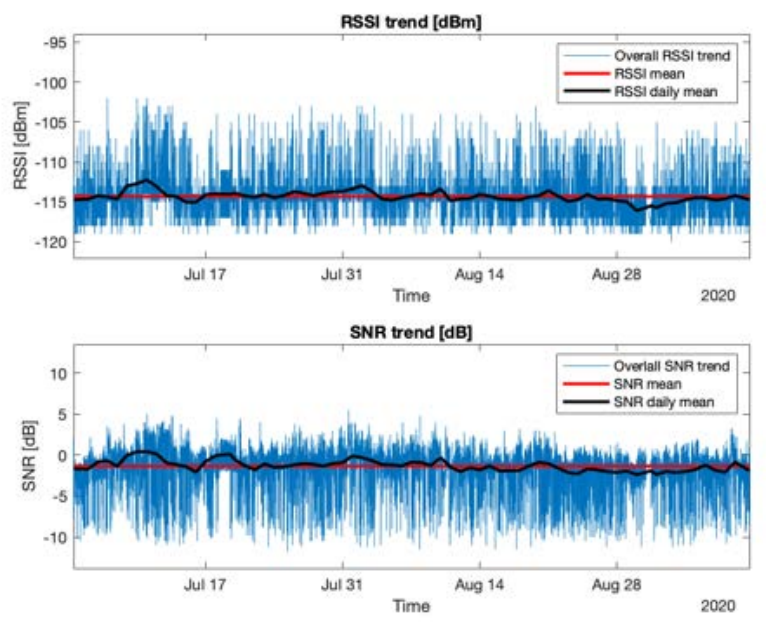

Fig. 8. Overall trend for RSSIs and SNRs along with total and daily means.

performances of LoRa transmission channel in sundry meteorological conditions can be drawn. Indeed, during the data collection period, a wide range of different relative humidity values was experienced (i.e., from $10 \%$ to $100 \%$ ). The same occurred for atmospheric pressure, whose values were measured between $998 \mathrm{hPa}$ and $1026 \mathrm{hPa}$. Concerning the temperature, the measured range was notably shorter since the measurements took place only in summer (i.e., $14.6^{\circ} \mathrm{C} \div 35.3^{\circ} \mathrm{C}$ ): nevertheless, it is well known that the higher the temperature, the higher the thermal noise, thus SNR values tend to be lower. This means that, since $35.3^{\circ} \mathrm{C}$ can be assumed as one of the highest achievable temperature values for the deployment site, the transmission channel has been analyzed in worst case scenario.
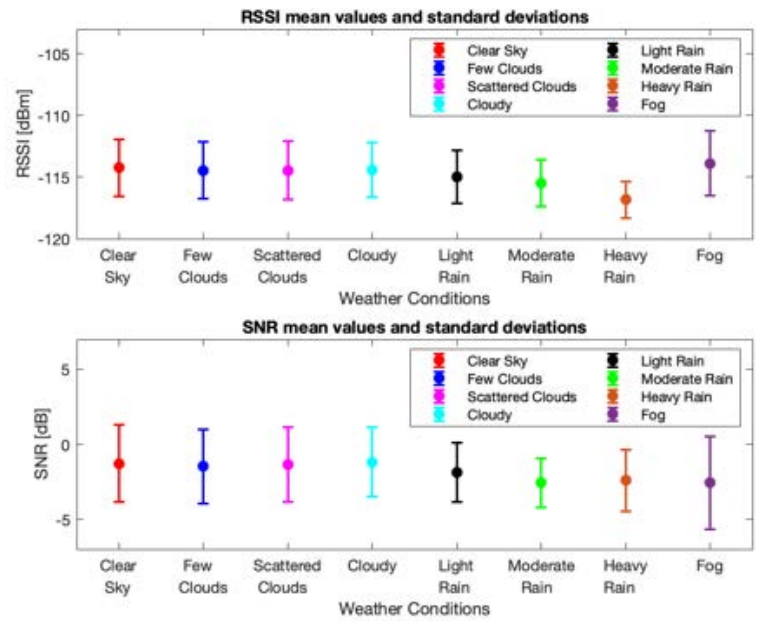

Fig. 9. RSSIs and SNRs mean values and standard deviations in function of weather conditions.

Tests results prove that weather affects transmission performances. Indeed, across the testing period, a wide range of different weather conditions was experienced, from clear sky to heavy rain and fog. Unfortunately, all of them were experienced during the same season (i.e., summer). Therefore, future works foresee to further extend the measurement campaign so as to include additional weather and seasonal conditions since different performances are expectable. Concerning summer, it is clearly visible from Fig. 9 that performances worsen in case of rain. As it will be mentioned below, this is in accordance with the signal degradation that is noticeable at high relative humidity levels and makes this parameter the only one that can significantly affect the actual transmission capabilities for the LoRa technology. Performances during fog show a strange behavior since the maximum mean value for RSSI was experienced. Such a fact is an oddity because fog is an aerosol made of tiny water droplets. However, this has a limited significance since only 8 samples were collected owing to the fact that in the deployment scenario fog is infrequent in summer. The restricted importance of this result is also supported by the fact that it has the biggest standard deviation. Another interesting phenomenon that can be noticed from Fig. 9 is that the performances are slightly better with cloudy sky than with clear sky: this could be due to the fact that clouds may act as a sort of waveguide, leading to a smaller signal dispersion.

Fig. 10 and 11 respectively report within their charts RSSIs and SNRs mean values and standard deviations in function of temperature, relative humidity and atmospheric pressure in comparison with the overall mean values and standard deviations computed throughout the whole measurement campaign. Despite the trends of RSSIs and SNRs mean values with respect to temperature slightly move away from the overall mean, they show an interesting behaviour: although the higher the temperature, the stronger the thermal noise effect, RSSIs and SNRs mean values increase with temperature. It is plausible to conclude that such a fact is the result of the superimposition of two effects taking place outdoor: low 


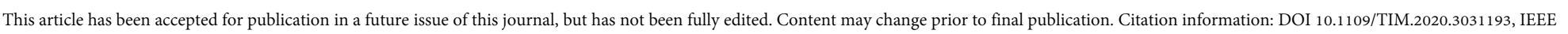
Transactions on Instrumentation and Measurement
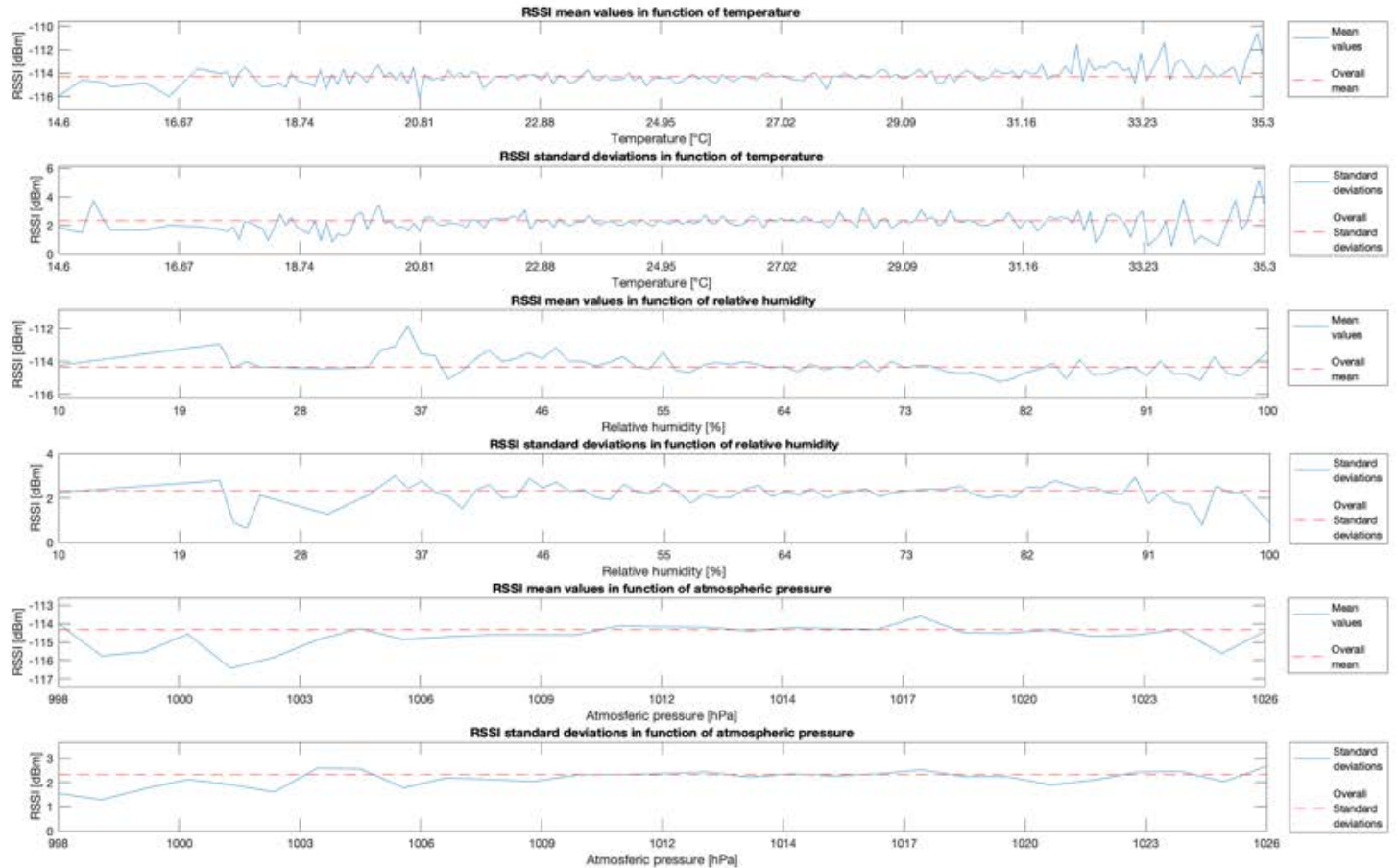

Fig. 10. RSSIs mean values and standard deviations in function of temperature, relative humidity and atmospheric pressure.
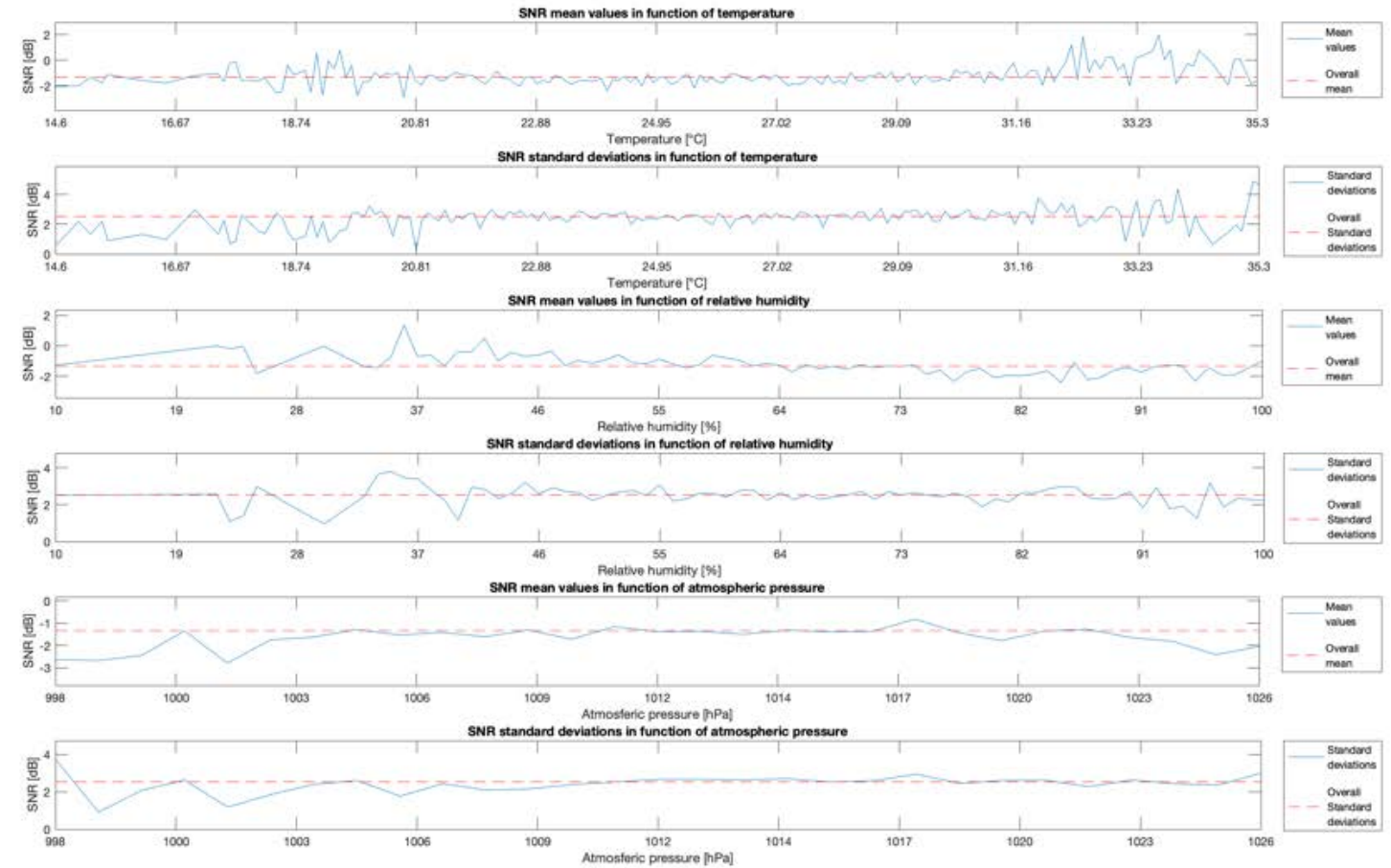

Fig. 11. SNRs mean values and standard deviations in function of temperature, relative humidity and atmospheric pressure. 
temperatures usually occur along with adverse meteorological conditions (e.g., moisture and rain) whose detrimental result has a more prominent extent than the beneficial one owing to low temperatures. As a result, RSSIs and SNRs tend to diminish in such situations. Hence, a similar phenomenon is likely to occur during winter were temperatures are much lower and weather is harsher. Moreover, RSSIs and SNRs are prone to decrease as relative humidity increases, respectively in the order of $2-3 \mathrm{dBm}$ for RSSI and $1-2 \mathrm{~dB}$ for SNR. This is foreseeable since the higher the relative humidity, the denser water vapour in air. This fact was confirmed during field tests even though a modest spike at maximum relative humidity was experienced: it has a little statistical relevance since a limited number of samples for maximum relative humidity were collected. Finally, atmospheric pressure plays a minor role since on the whole no significant performances variation can be noticed, apart from a slight decline for low pressure values.

\section{CONCLUSION}

IoT infrastructures are becoming common day after day in the most diverse industrial fields. In this paper, an IoT monitoring infrastructure to be used for the remote control of offshore sea farms was presented: the proposed system is currently deployed on site offshore the city of Piombino, Italy. Due to the harshness of the deployment scenario (i.e., in the middle of the sea), at a distance of some kilometers from the sea shore (i.e., $8.33 \mathrm{~km}$ ), an ad hoc network infrastructure based on the LoRaWAN protocol was developed and tested. The launch of the seamark buoy hosting the instrumentation allowed to test the transmission performances by analyzing variation related to sundry meteorological parameters (i.e., temperature, relative humidity and atmospheric pressure) and weather conditions. The RSSI and SNR values presented in this work allow to state that the LoRa technology is extremely robust: in particular, only relative humidity, as well as the presence of rain, proved to have some disruptive effect on the transmission channel. Nevertheless, the reduction of the transmission parameters is very low (i.e., in the order of 2-3 dBm for RSSI and 1-2 $\mathrm{dB}$ for SNR), suggesting that these parameters may become crucial only for very long transmission ranges.

\section{REFERENCES}

[1] M. Kobayashi, S. Msangi, M. Batka, S. Vannuccini, M.M. Dey and J. L. Anderson, Fish to 2030: the role and opportunity for aquaculture. Aquaculture economics \& management, 19(3), pp. 282-300, 2015.

[2] A. Fredheim and T. Reve, Future Prospects of Marine Aquaculture. In Proceedings of OCEANS 2018 MTS/IEEE Charleston, Charleston, SC, USA, 22-25 October 2018, pp. 1-8.

[3] L. Parri, S. Parrino, G. Peruzzi and A. Pozzebon, Low Power Wide Area Networks (LPWAN) at Sea: Performance Analysis of Offshore Data Transmission by Means of LoRaWAN Connectivity for Marine Monitoring Applications. Sensors, 19(14), 3239, 2019.

[4] L. Parri, S. Parrino, G. Peruzzi and A. Pozzebon, A LoRaWAN Network Infrastructure for the Remote Monitoring of Offshore Sea Farms. In Proceedings of 2020 IEEE International Instrumentation and Measurement Technology Conference (I2MTC), Dubrovnik, Croatia, 25-28 May 2020, pp. 1-6.
[5] T. Addabbo, A. Fort, M. Mugnaini, L. Parri, S. Parrino, A. Pozzebon and $\mathrm{V}$. Vignoli, An IoT framework for the pervasive monitoring of chemical emissions in industrial plants. In Proceedings of 2018 Workshop on Metrology for Industry 4.0 and IoT, Brescia, Italy, 16-18 April, pp. 269273.

[6] D. Davcev, K. Mitreski, S. Trajkovic, V. Nikolovski and N. Koteli, N. IoT agriculture system based on LoRaWAN. In Proceedings of 2018 14th IEEE International Workshop on Factory Communication Systems (WFCS), Imperia, Italy, 13-15 June 2018, pp. 1-4.

[7] M. Cerchecci, F. Luti, A. Mecocci, S. Parrino, G. Peruzzi and A. Pozzebon, A low power IoT sensor node architecture for waste management within smart cities context. Sensors, 18(4), 1282, 2018.

[8] K. S. Duisebekova, Z. N. Sarsenova, V. T. Pyagay, Z. N. Tuyakova, N. T. Duzbayev, A. Z. Aitmagambetov and S. T. Amanzholova, Environmental monitoring system for analysis of climatic and ecological changes using LoRa technology. In Proceedings of the 5th International Conference on Engineering and MIS, Astana, Kazakhstan, 6-8 June 2019, p. 15.

[9] T. P. Truong, L. M. Nguyen, T. H., Le and B. Pottier, Study on Long Range Radio Communication for Environmental Monitoring Applications. In Proceedings of the 2019 2nd International Conference on Electronics, Communications and Control Engineering, Phuket, Thailand, 13-15 April 2019, pp. 92-97.

[10] L. Lombardo, M. Parvis, E. Angelini and S. Grassini, An Optical Sampling System for Distributed Atmospheric Particulate Matter. IEEE Transactions on Instrumentation and Measurement, 68(7), pp. 2396-2403, 2019.

[11] J. Botero-Valencia, L. Castano-Londono, D. Marquez-Viloria and M. Rico-Garcia, Data reduction in a low-cost environmental monitoring system based on LoRa for WSN. IEEE Internet of Things Journal, 6(2), pp. 3024-3030, 2018

[12] D. Madeo, A. Pozzebon, C. Mocenni and D. Bertoni, A Low-Cost Unmanned Surface Vehicle for Pervasive Water Quality Monitoring. IEEE Transactions on Instrumentation and Measurement, 69(4), pp. 1433-1444, 2020.

[13] M. Cattani, C. A. Boano and K. Romer, An experimental evaluation of the reliability of lora long-range low-power wireless communication. Journal of Sensor and Actuator Networks, 6(2), 7, 2017.

[14] R. Sanchez-Iborra, J. Sanchez-Gomez, J. Ballesta-Vinas, M. D. Cano and A. F. Skarmeta, Performance evaluation of LoRa considering scenario conditions. Sensors, 18(3), 772, 2018.

[15] E. D. Ayele, C. Hakkenberg, J. P. Meijers, K. Zhang, N. Meratnia and P. J. Havinga, Performance analysis of LoRa radio for an indoor IoT applications. In Proceedings of 2017 International Conference on Internet of Things for the Global Community (IoTGC), Funchal, Portugal, 10-13 July 2017, pp. 1-8.

[16] A. Rahman and M. Suryanegara, The development of IoT LoRa: A performance evaluation on LoS and Non-LoS environment at $915 \mathrm{MHz}$ ISM frequency. In Proceedings of 2017 International Conference on Signals and Systems (ICSigSys), Sanur, Indonesia, 16-18 May 2017, pp. 163-167.

[17] S. Y. Wang, Y. R. Chen, T. Y. Chen, C. H. Chang, Y. H. Cheng, C. C. Hsu and Y. B. Lin, Y. B. Performance of LoRa-based IoT applications on campus. In Proceedings of 2017 IEEE 86th Vehicular Technology Conference (VTC-Fall), Toronto, ON, Canada, 24-27, September 2017 , pp. 1-6

[18] J. Haxhibeqiri, A. Karaagac, F. Van den Abeele, W. Joseph, I. Moerman and J. Hoebeke, LoRa indoor coverage and performance in an industrial environment: Case study. In 2017 22nd IEEE international conference on emerging technologies and factory automation (ETFA), Limassol, Cyprus, 12-15 September 2017, pp. 1-8.

[19] T. Petric, M. Goessens, L. Nuaymi, L. Toutain and A. Pelov, Measurements, performance and analysis of LoRa FABIAN, a real-world implementation of LPWAN. In Proceedings of 2016 IEEE 27th Annual International Symposium on Personal, Indoor, and Mobile Radio Communications (PIMRC), Valencia, Spain, 4-8 September 2016, pp. 1-7.

[20] A. Augustin, J. Yi, T. Clausen and W. M. Townsley, A study of LoRa: Long range \& low power networks for the internet of things. Sensors, 16(9), 1466, 2016.

[21] D. Yim, J. Chung, Y. Cho, H. Song, D. Jin, S. Kim, S. Ko, A. Smith and A. Riegsecker, An experimental LoRa performance evaluation in tree farm. In 2018 IEEE sensors applications Symposium (SAS), Seoul, South Korea, 12-14 March 2018, pp. 1-6.

[22] L. Vangelista, A. Zanella and M. Zorzi, Long-range IoT technologies: The dawn of $\mathrm{LORa}^{T M}$. Future access enablers of ubiquitous and intelligent infrastructures, 159, 51-58, 2015. 
[23] F. Adelantado, X. Vilajosana, P. Tuset-Peiro, B. Martinez, J. Melia-Segui and T. Watteyne, Understanding the limits of LoRaWAN. IEEE Commun. Mag., 55(9), 34-40, 2017.

[24] P. Jörke, S. Böcker, F. Liedmann and C. Wietfeld, Urban channel models for smart city IoT-networks based on empirical measurements of LoRa-links at 433 and $868 \mathrm{MHz}$. In Proceedings of the IEEE 28th Annual International Symposium on Personal, Indoor, and Mobile Radio Communications (PIMRC), Montreal, Canada, 8-13 October 2017, pp. $1-6$.

[25] L. K. A. Terashmila, T. Iqbal and G. Mann, A comparison of low cost wireless communication methods for remote control of grid-tied converters. In Proceedings of the IEEE 30th Canadian Conference on Electrical and Computer Engineering (CCECE), Windsor, Canada, 30 April-3 May 2017, pp. 1-4.

[26] M. Pulpito, P. Fornarelli, C. Pomo, P. Boccadoro and L. A. Grieco, On fast prototyping LoRaWAN: a cheap and open platform for daily experiments. IET Wirel. Sens. Syst., 8(5), 237-245, 2018.

[27] Y. Cui, H. Liu, M. Zhang, S. Stankovski, J. Feng and X. Zhang, X. Improving Intelligence and Efficiency of Salt Lake Production by Applying a Decision Support System Based on IOT for Brine Pump Management. Electronics, 7(8), 147, 2018.

[28] F. Agbuya, G. F. Apolinario, D. M. Ramos, J. M. Villanueva, P. Zafe, J. A. Hernandez and J. Coquia, Design of a Real-Time Ocean Data-Logging Drifter Thru CLOUD Technology for Collecting Tidal Parameters. In Proceedings of TENCON 2018-2018 IEEE Region 10 Conference, Jeju, South Korea, 28-31 October 2018, pp. 0844-0849.

[29] R. Gerin, M. Zennaro, M. Rainone, E, Pietrosemoli, P. A. Poulain and A. Crise, On the design of a sustainable ocean drifter for developing countries. EAI Endorsed Transactions on Internet of Things, 2019.

[30] N. Jovalekic, V. Drndarevic, E. Pietrosemoli, I. Darby and M. Zennaro, Experimental study of LoRa transmission over seawater. Sensors, 18(9), 2853, 2018.

[31] STMicroelectronics, STM32L073x8, STM32L073xB and STM32L073xZ datasheet, October 2019. Available online: https://www.st.com/resource/en/datasheet/stm321073v8.pdf (accessed on 8 July 2020).

[32] HopeRF,RFM95W/96W/98W datasheet, July 2019. Available online: https://www.hoperf.com/data/upload/portal/20190801/RFM95W-V2. 0 .pdf (accessed on 8 July 2020).

[33] Semtech, SX1276/77/78/79 datasheet, January 2019. Available online: https://www.semtech.com/uploads/documents/DS_SX1276-7-8-9_W_ APP_V6.pdf (accessed on 12 August 2019).

[34] LoRa Alliance, LoRaWAN1.0.3 Regional Parameters, July 2018. Available online: https://lora-alliance.org/sites/default/files/2018-07/lorawan_ regional_parameters_v1.0.3reva_0.pdf (accessed on 8 July 2020).

[35] Rak, RAK831 Lora Gateway Datasheet V1.3, 2017. Available online: https://downloads.rakwireless.com/en/LoRa/RAK831-LoRa-Gateway/ Hardware-Specification/RAK831\%20Datasheet\%20V1.3.pdf(accessed on 12 August 2019).

[36] Semtech, SX1257 Low Power Digital I and Q RF Multi-PHY Mode Transceiver datasheet, March 2018. Available online: https://www. semtech.com/uploads/documents/DS_SX1257_V1.2.pdf (accessed on 12 August 2019).

[37] Semtech, SX1301 datasheet, June 2017. Available online: https:// www.semtech.com/uploads/documents/sx1301.pdf (accessed on $12 \mathrm{Au}-$ gust 2019)

[38] OpenWeather. Available online: https://openweathermap.org (accessed on 22 July 2020).

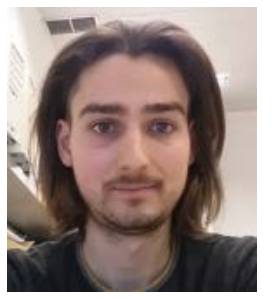

Lorenzo Parri received the MSc in Electronics and Communications Engineering from the University of Siena, Italy, in 2017 . He is currently a PhD student in Information Engineering at the Department of Information Engineering and Mathematics of the University of Siena. His current research interests include the design of exhausts gas measurement systems, electronic systems for industrial application and condition monitoring and the design of embedded microcontroller-based devices.

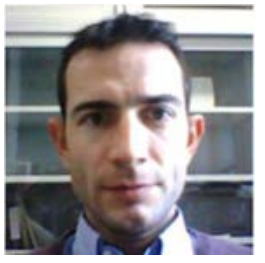

Stefano Parrino received the MSc in Information Engineering, with a focus on Radio Frequency Identification (RFID) technology applied to healthcare environments, in 2008, from the University of Siena, Italy. He is currently a Research Fellow at the Department of Information Engineering and Mathematics, University of Siena. His main research focuses on RFID applications and on design and development of embedded monitoring systems based on Low-Power Wide Area Networks (LPWANs), microapplied in different application fields.

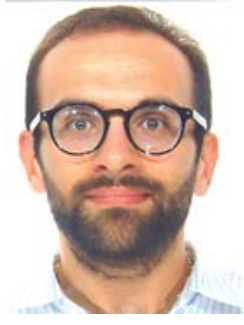

Giacomo Peruzzi received the BSc in Information Engineering, in 2016, and the MSc in Computer and Automation Engineering, in 2019, from the University of Siena, Italy. He is currently a $\mathrm{PhD}$ student in Information Engineering at the Department of Information Engineering and Mathematics of the University of Siena. His research activity principally falls within the fields of the Internet of Things (IoT) and Industry 4.0. In particular, he deals with Wireless Sensor Networks (WSNs) for monitoring systems which are enabled by Low-Power Wide Area Network (LPWAN) technologies.

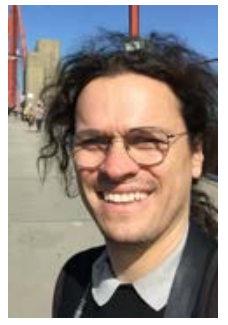

Alessandro Pozzebon (Member, IEEE) received the MSc in information engineering, with a focus on the radio frequency identification (RFID) technology applied to cultural heritage, and the $\mathrm{PhD}$ from the University of Siena, Siena, Italy, in 2006 and 2012, respectively. His PhD thesis was on the use of RFID technology for environmental monitoring of coastal erosion phenomena. He is currently an Assistant Professor with the Department of Information Engineering and Mathematics, University of Siena, where he teaches in the Laboratory of Internet of Things. His main research interests include the development of applications based on Low-Power Wide Area Network (LPWAN), Wireless Sensor Network (WSN), and RFID technologies in several different application fields, from healthcare to cultural heritage and environmental monitoring. 